\title{
Courrier au BMS
}

\section{Antwort auf den offenen Brief der Präsidentin des Bündner Ärzte- vereins, Frau Dr. H. Jörimann}

Mehr Qualität und Selbstbestimmung bei medizinischen Eingriffen

Ärzte lassen an sich oder ihren Familienmitgliedern nachweislich gewisse Eingriffe signifikant weniger häufig durchführen als an ihren Patienten. Wieso wurde Frauen aus der Unterschicht überdurchschnittlich oft die Gebärmutter entfernt? Wieso gibt es heute noch unzählige unnötige Eingriffe gegen Prostatakrebs, die zu schweren Beeinträchtigungen der Lebensqualität führen, obwohl diese Diagnose zu Lebzeiten oft gar keinen Schaden anrichten kann? Niemand geringerer als der Entdecker des Prostata-spezifischem Antigens (PSA), Richard Ablin, spricht von einer "profitgetriebenen Katastrophe für das Gesundheitssystem». Ähnliche Kritik gibt es gegen Massenscreenings zu Brustkrebs, die oft mehr Angst und Schaden anrichten als diese Nutzen generieren. Die Kantone Bern und Solothurn wollen nun deshalb die Notbremse ziehen. «Fragwürdige Weltmeisterin" ist die Schweizer Chirurgie übrigens bei der Implantierung von Knie- und Hüftgelenken und dies obwohl weniger weitgehende Eingriffe häufig bessere Resultate erzielen.

\section{Mehr Selbstbestimmung, weniger \\ Bevormundung}

Nur wenn der Patient zum Voraus über die tatsächlichen Chancen und Risiken sowie die Alternativen in verständlicher Weise aufgeklärt wird, hat er eine echte Chance auf Selbstbestimmung. Faktisch liegt oft eine subtile Steuerung durch das Spital oder den behandelnden Arzt vor. Sicherlich wollen unsere Ärztinnen und Ärzte in der Regel das Beste für ihre Patientinnen und Patienten; trotzdem bleibt festzuhalten: Betriebsblindheit gibt es auch bei diesen Berufsleuten - und diese wird nicht eben gebremst, wenn an jedem Eingriff verdient wird, selbst wenn er überflüssig oder gar kontraproduktiv ist. Sich im Spital oder beim Arzt wohl zu fühlen ist daher nicht genug! Die Qualität eines Eingriffs ist der Unterschied von vor der Behandlung zu nachher - und dieser Unterschied ist messbar! Wenn unsere Bevölkerung kaufkraftbereinigt mehr als doppelt so viel Geld für die Gesundheit ausgibt wie in Italien oder Spanien, muss dies ergebnisbezogen auch wesentlich deutlicher zum Ausdruck kommen - was jedoch leider nicht der Fall ist! Es braucht deshalb dringend mehr Transparenz bei der «Ergebnis- qualität»: Welche gesundheitliche Veränderung hat ein Eingriff in einer bestimmten Situation für einen Patienten gebracht? Nur wenn Betroffene und Angehörige umfassend und verständlich aufgeklärt werden über den Mehrwert eines medizinischen Eingriffs, können diese persönlich auch die für sie richtigen Entscheide treffen. Solange dies nicht der Fall ist, bleibt gültig, was Bundesrat und Gesundheitsexperten belegen, auch wenn es nicht gerne gehört wird: 20 Prozent der medizinischen Eingriffe sind überflüssig! Sie haben für die $\mathrm{Pa}$ tientinnen und Patienten keinen Mehrwert, sondern für diese nur Belastungen zur Folge punkto Gesundheit und letztlich auch beim Portemonnaie bzw. den Krankenkassenprämien.

\section{Effizienz und Qualität statt Rationierung} Ich bin mit der Präsidentin des Bündner Ärztevereins aber insofern einig, dass wir unserer Bevölkerung keine Rationierung zumuten dürfen und wollen. Jedermann soll im Bedarfsfall rasch die notwendige und wirksame medizinische Versorgung erhalten. Damit dies so bleibt, muss systematisch in die Effizienz und in die Qualität investiert werden: Wir wollen bei den medizinischen Behandlungen nicht in die zweite Liga absteigen, aber bei den Eintrittspreisen jene der Champions League bezahlen.

Heinz Brand

Präsident von santésuisse und Nationalrat SVP

\section{Hinterfragen und erweitern}

Brief zu: Adler R. Credo Quia Absurdum - oder: Sparmöglichkeiten im Gesundheitswesen. Schweiz Ärztezeitung 2017:98(28-29):901-2.

Geschätzter Herr Kollege Prof. em. Dr. med. R. Adler

Sie und der von Ihnen zitierte Edzard Ernst haben völlig Recht. Berliner Mauer ohne genaue Analyse der Zusammensetzung des verwendeten Mauerstücks und ohne eine korrekt durchgeführte homöopathische Arzneimittelprüfung therapeutisch einzusetzen, widerspricht den Regeln der ärztlichen Homöopathie.

Wenn wir Ärztinnen und Ärzte aber plötzlich verantwortlich wären für alle Medikamente, die hergestellt werden, wo führte das hin? Müsste man die Methode als Ganzes in Verruf bringen, wenn z.B. ein Medikament wegen nachgewiesenen, starken Nebenwirkungen vom Markt genommen werden muss? Ist die Rheumatologie als Methode falsch wegen dem Fiasko mit den COX-2-Hemmern?

Sie führen unwissenschaftliches Denken auf das Medizinstudium zurück, das Fakten vermittelt statt wissenschaftlichem Denken. Sie können sich freuen, denn mit dem neuen Lernzielkatalog «PROFILES» (= Principal Relevant Objectives and Framework for Integrated Learning and Education in Switzerland), der am 15.3.17 definitiv genehmigt wurde durch SMIFK/CIMS wurde genau diesem Umstand Rechnung getragen. Der neue Lernzielkatalog beinhaltete einen Paradigmenwechsel weg vom reinen Vermitteln von Wissen hin zu einem kompetenzbasierten Handeln. Interessant z.B. GO 6.4: «understand the general theoretical principles of medical and scientific knowledge and show an awareness of its development, its problems and limits». Was heisst, dass man das heute gültige naturwissenschaftliche Denken in der Medizin, das übrigens immer noch auf den Prinzipien von Newton beruht, durchaus hinterfragen und auch erweitern darf!

Und ausserdem bezüglich Komplementärmedizin: EPA 7.3 "Adopt a shared-decision making approach in establishing the management plan, take into account patients' preferences in making orders; take into account an indication or request for complementary medicine; (...). »

Der Schweizer Verein homöopathischer Ärztinnen und Ärzte setzt sich ein für eine integrative Homöopathie zum Nutzen vieler Patientinnen und Patienten und hat als Mitglied der UNION Schweizerischer komplementärmedizinischer Ärzteorganisationen letztes Jahr die Qualitäts-Charta der FMH unterzeichnet zusammen mit vielen anderen Ärztevereinigungen.

Dr. med. Gisela Etter, Präsidentin Schweizer Verein homöopathischer Ärztinnen und Ärzte (SVHA), Richterswil

\section{Lettres de lecteurs}

Envoyez vos lettres de lecteur de manière simple et rapide. Sur notre site Internet, vous trouverez un outil spécifique pour le faire. Votre lettre de lecteur pourra ainsi être traitée et publiée rapidement. Vous trouverez toutes les informations sous:

www.bullmed.ch/auteurs/

envoi-lettres-lecteurs/ 
Certificat laboratoire du praticien selon régime transitoire

Message envoyé au KHM CMPR

Bonjour,

j'ai bien reçu votre facture Nr 14'306 du 6.7.2017 et vous signale que je ne la payerai pas: je n'ai pas souvenir d'avoir demandé un «Certificat laboratoire du praticien selon régime transitoire», et même si peut-être, je l'ai fait, j'ai changé d'avis et désire mettre à votre disposition, ainsi que du Comité Central de la FMH et du Courrier des lecteurs du BMS, mes considérations suivantes:

1. Depuis l'obtention de mon titre de Pédiatre FMH en 1984, j'ai introduit, soit comme praticien et consultant dans 2 hôpitaux de district, soit comme médecin-cadre et médecin-chef dans 2 cliniques de pédiatrie, la bandelette urinaire et le frottis rapide à la recherche de streptocoques. Je ne compte pas les douzaines de médecins-assistants, assistantes médicales et autres professionnels auxquelles j'ai enseigné la théorie, la pratique et les pièges de ces 2 tests. 2. Bien sur que les experts, et les autorités qui s'appuient sur eux, ont pris en considération qu'en introduisant ce "Certificat laboratoire du praticien selon régime transitoire», ils désavouent la quarantaine de sites de formation en pédiatrie, et les programmes de formation que l'on nous a imposés de mettre en place (cf. chiffres 7.3.,8.1. et 8.2 des annexes du règlement correspondant): Si la formation est accomplie et l'examen (de spécialiste en Pédiatrie) réussi, un "Certificat laboratoire du praticien selon régime transitoire» (pour tremper une bandelette dans de l'urine correctement récolté) ou frotter une amygdale est superflu, et de l'exiger constitue à mon avis une chicane ridicule.

3. Les mêmes experts et les autorités ont bien sûr pris en considération une période de transition: ont-ils calculé combien de test un spécialiste réduisant progressivement son activité, afin de garantir à ses malades une transition harmonieuse, sans frais gris, à ses successeurs, devrait encore facturer,pour que les 300 francs exigés pour un papier du moins discutable (vide 2.) ne constituent pas une pure perte?

4. Bref, les frottis de gorge et urines de mes malades devront donc être transportés au laboratoire de l'hôpital, et les traitements gérés par téléphone: heureusement que l'on a prévu de nous limiter à 20 minutes/trimestre, car les mêmes experts et les autorités savent pertinemment que les petits enfants ne sont pas autorisés à extérioriser plus 4 poussées fébriles inexpliquées par année. Bien sûr que si dans l'intervalle, les autorités ont prévu que les pédiatres et autres médecins de premier recours peuvent facturer les tests dits de «bed-side-diagnosis» les plus fréquents tels que la bandelette urinaire et le frottis rapide à la recherche de streptocoques sans certificat, je retire mes critiques à cet égard.

\section{Cordiales salutations}

Dr méd. Alexandre Corboz, Pédiatre, FMH Spéc. gastroentérologie pédiatrique DIU d'endoscopie digestive et proctologie pédiatrique Médecin-consultant au Département de Pédiatrie de l'HNE

\section{Réplique}

Lettre concernant Corboz A. Certificat laboratoire du praticien selon régime transitoire. Bull med. suisses 2017;98(32):992.

\section{Cher Collègue,}

Nous faisons suite à votre courrier de lecteur et pouvons apporter quelques éclaircissements à vos réflexions, que partagent bon nombre de praticiens.

S'agissant de votre dossier personnel, nous vous invitons à prendre contact avec notre secrétariat. Nous tenons ici à relever qu'il arrive fréquemment que les cabinets de groupes ou hôpitaux fassent des demandes groupées pour l'ensemble des médecins pratiquant le laboratoire dans leur établissement. Il se peut donc que votre employeur ait fait une demande en votre nom en ce sens. L'AFC-LP étant personnelle, les factures sont adressées au nom du médecin requérant le diplôme de formation postgraduée.

\section{Historique de l'AFC-LP (extrait et adaptation} de Paediatrica vol. 27 No 52016 p. 9):

Dans les années 1990, le laboratoire du praticien était soumis à de fortes pressions de la part des laboratoires privés et de l'OFSP, arguant que la qualité du laboratoire médical était insuffisante et ne correspondait pas aux normes ISO.

La Commission suisse pour l'assurance de qualité dans le laboratoire médical (QUALAB) a été créée en 1994, sur la base de la Loi fédérale sur l'assurance maladie. Elle est responsable de l'élaboration et de la réalisation des mesures nécessaires au contrôle qualité. Des contrôles qualité externes et internes obligatoires ont été introduits dans ce cadre.

Font partie des critères de qualité d'un laboratoire médical une bonne formation postgraduée en analyses médicales du responsable du laboratoire ainsi qu'une connaissance des bases légales et économiques. Les connaissances en analyse médicale acquises par les médecins en formation postgraduée étant très variables, il a été exigé qu'une formation postgraduée uniforme soit développée dans ce domaine. La FMH a été contrainte de se soumettre à cette exigence. Elle a délégué la création de l'attestation de formation complémentaire pour la pratique du laboratoire au cabinet médical (AFC-LP) au Collège de médecine de premier recours CMPR, lequel a mis sur pied une commission composée de médecins de famille et de professionnels de laboratoires, afin d'élaborer un programme de formation complémentaire. Ce programme est entré en vigueur en 2001, mais le caractère obligatoire de l'AFC-LP a pu être retardé jusqu'en 2017.

L'Union suisse de médecine de laboratoire (USML) a été chargée par QUALAB de revoir les critères de fonctionnement des laboratoires d'analyses médicales (CFLAM) et de les adapter aux normes internationales ISO 15189:2012 «Laboratoires médicaux - exigences de qualité». QUALAB, les assureurs et l'Office fédéral de la santé publique poussaient depuis des années pour que la formation postgraduée en responsable de laboratoire soit uniformisée et déclarée obligatoire. En date du 16.03.2016, l'assemblée des délégués de la FMH a accepté l'obligation de l'AFC-LP à une grande majorité. Suite à la révision de la CFLAM, l'acquisition de l'AFC-LP du CMPR est donc devenue obligatoire dès le $1^{\text {er }}$ janvier 2017 pour tous les médecins qui souhaitent facturer aux assureurs des prestations de laboratoire effectuées dans leur propre laboratoire médical. Afin de faciliter l'obtention de l'attestation, une disposition transitoire a été introduite jusqu'au 31 décembre 2017, laquelle permet aux médecins ayant effectué et facturé, pendant au moins 2 ans avant l'introduction du règlement obligatoire, des prestations de laboratoire dans un laboratoire de cabinet médical participant aux contrôles de qualité externes, d'obtenir l'AFCLP sans avoir à participer au cours. Une fois l'attestation obtenue, les contrôles réguliers de la qualité du laboratoire remplacent le renouvellement du certificat.

\section{En conclusion}

Il existe des situations discutables dans toute réglementation, mais il ne faut pas oublier que, d'une part, la solution actuelle constituait le seul compromis politique possible pour sauver le laboratoire du praticien et que, d'autre part, le rôle du médecin dans ces gestes de laboratoire est plus complexe qu'il n'y paraît, puisque le résultat brut doit être interprété dans son contexte clinique. Le thème de la bandelette urinaire - qui peut au demeurant être achetée dans le commerce et réalisée par les patients eux-mêmes - n'est donc pas représentatif de la problématique.

Nous vous prions de croire, cher Collègue, à l'assurance de nos salutations confraternelles.

Pierre Klauser, Président du CMPR 


\section{Pourquoi tant de secrets à propos du tarif proposé par M. Berset?}

Lettre à propos de: Müller P. Exercice d'économie du Conseil fédéral et soins ambulatoires. Bull Méd Suisses. 2017;98(888-91).

Enfin un article un peu plus explicatif, donc meilleur, sur le problème du tarif "proposé» par le Conseil fédéral. Il y a incontestablement progrès. Mais comme toujours, et ce n'est pas la première fois que je me plains, une/des comparaison(s) sont incomplètes, et juste destinées à nous émouvoir, sans nous décrire l'ensemble de la situation.

Le graphique proposé en page 890 du BMS ne comporte pas de légendes claires le long des axes. On comprend pour les abcisses, mais la légende des ordonnées est «caviardée». De plus on ne dit pas combien de médecins seraient touchés par une ligne de réduction calculée, donc l'effectif de chaque groupe en ligne, ni combien ils gagnent en moyenne actuellement pour chaque ligne calculée! On exprime une "moyenne», mais on ne sait pas avec quoi ni comment on a calculé cette moyenne générale. Pourquoi nous proposer cela, qui ne fait pas comprendre les enjeux de manière claire. Un problème avec la "confraternité»?

Quand on veut que tout le monde agisse ensemble, on rate la cible, et cela ressort de la manipulation. A l'OFSP on est certainement pas assez naïf pour avaler cela! Et ils ont les chiffres complets !

Dr Virgile Woringer, Lausanne

\section{Réponse}

Avec ce graphique, nous avons voulu montrer que toutes les disciplines sont concernées par la deuxième intervention tarifaire. L'impact de l'intervention a été simulé sur la base de notre transcodage et du volume de prestations de chaque discipline selon NewIndex. Pour cela, nous avons volontairement flouté les différentes disciplines le long de l'axe y (code NAKO). Nous avons simulé l'impact en détail pour chaque discipline et leur avons mis les résultats à disposition. Les chiffres représentent une moyenne (de tous les médecins d'une discipline) par rapport au nombre total de points tarifaires. Il est tout à fait possible que dans une discipline donnée, l'impact de l'intervention soit totalement différent d'un médecin à l'autre selon sa gamme de prestations.

Patrick Müller, Chef de division Médecine et tarifs ambulatoires

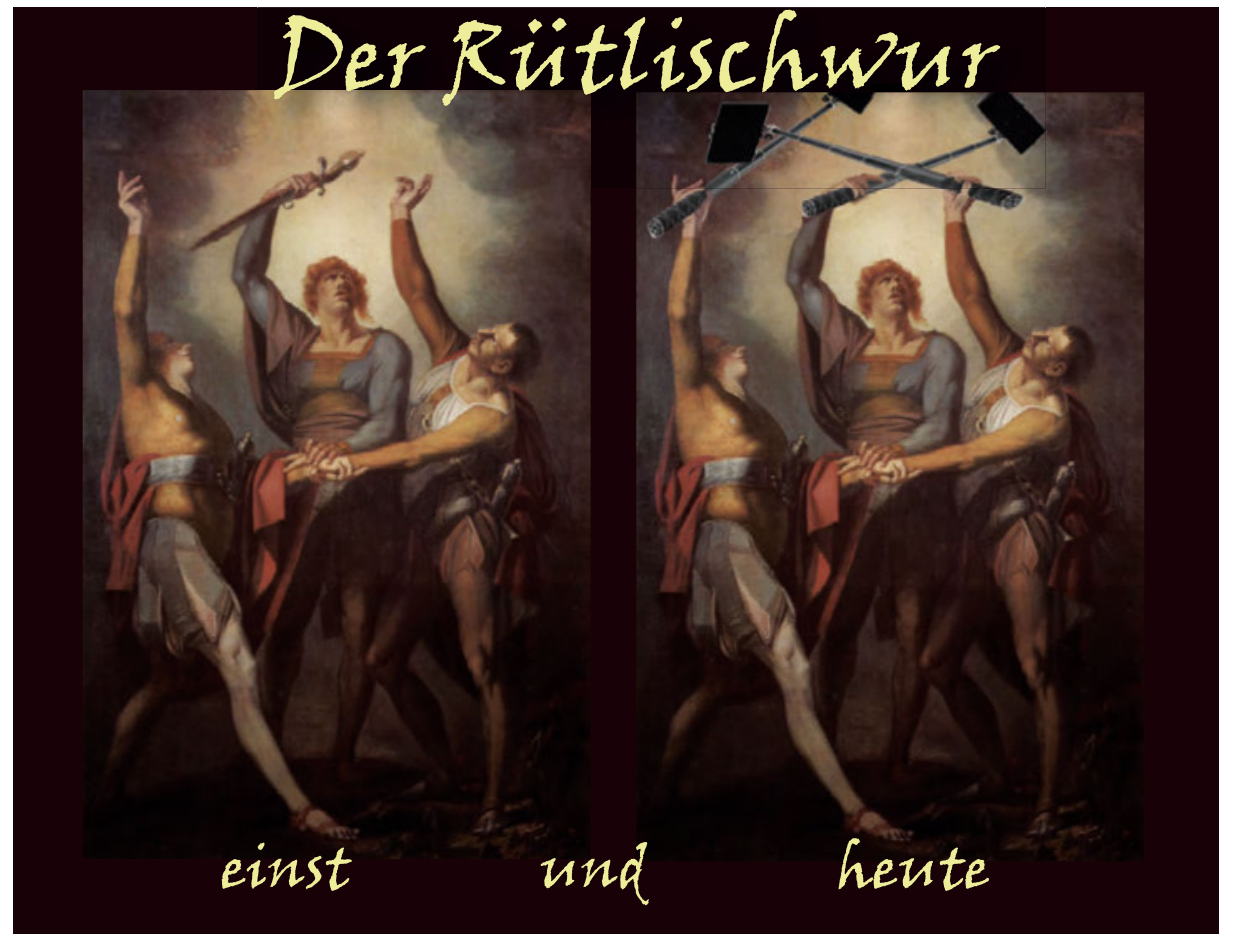

\section{Versicherungsmedizin}

Die Autoren Mast und Schneuwly, ein Neurologe und ein Kommunikationsspezalist, werben für mehr Verständnis für die Versicherungsmedizin [1]. Dabei wird Missverständnis und Unverständnis für die Fachkompetenz und Tätigkeit von Psychiater(inne)n und den von uns behandelten Störungen sichtbar.

Die Autoren beginnen ihre Stellungnahme mit den Honoraren. Dabei ist ihnen offenbar nicht bewusst, dass einerseits die Versicherungsmedizin von uns Behandlern eine "Bringschuld" an diagnostischen Befunden und fachkompetenter Beurteilung der Funktionseinschränkungen erwartet, andererseits eben diese Berichte von den auftraggebenden Versicherungen häufig mit nur einem geringen Honorarangebot von 40 bis 120 Franken einverlangt werden.

Von den Autoren wird auf eine «biologisch verstandene Gesundheitsstörung" verwiesen. Damit können auch unsere sehr sorgfältig geschriebenen Berichte als nichtig beurteilt werden. Alle sogenannten funktionellen und somatoformen Störungen sind betroffen und werden von der Versicherungsmedizin als «lediglich möglich» beurteilt. Es handelt sich allesamt um Störungen, gemäss ICD-10 klassifiziert. Die Tatsache, dass für viele der sogenannten "funktionellen Störungen» im klinischen Alltag noch keine in-vivo-menschenwürdig-anwendbare «biologische» Diagnostik vorliegt, wird den betroffenen Patienten zum Verhäng- nis. Es geht um Patienten, welche auch durch Nichtanerkennung der Invalidität nicht gesunder werden (siehe Untersuchung D. Brühlmeier, [2]).

Zwar liegen zahlreiche Hinweise aus der Grundlagenforschung vor (z.B. Gen-Demethylierungen bei Schmerzverarbeitungsstörungen, Veränderungen der Neuronalen Netzwerke durch Mangelerfahrungen in der kindlichen Entwicklung usw.). Wissen, welches keine Evidenz in der Klinik nachweist, wird nicht einbezogen. Es sei denn die IV selber wollte nicht evidente Untersuchungsmethoden anwenden, um «Lügner» zu ertappen. Dass neue naturwissenschaftliche Kenntnisse (siehe z.B. NZZ am Sonntag [3]) sich mit unserem Erfahrungswissen decken, aber die Lücke für die klinische Anwendung bisher nicht geschlossen werden konnte, wird offenbar von der Versicherungsmedizin nicht diskutiert.

Uns Psychiatern steht ein steht ein riesiges klinisches Erfahrungswissen zur Verfügung. Was für uns anerkannte Methoden, erlernt in 5- bis 6-jähriger Weiterbildung sind, wird von den mit unserer Fachkompetenz unvertrauten Kollegen als subjektiv und damit nicht verwendbar taxiert. Das Fach Psychiatrie und Psychotherapie hat gerade dort, wo die Medizin bisher nicht in der Lage war, die biologischen Grundlagen der Störungen zu erkennen, andere Ansätze der Diagnostik hervorgebracht (AMDP, operationalisierte psychodynamische Diagnostik OPD, usw.). Zudem wird ausgeblendet, dass auch die sogenann- 
ten biologischen Befunde fehlerhaft sein können und ebenso der Interpretation bedürfen. Somit sind diese nicht einfach "wahrer» als die von uns sorgfältig erhobenen Befunde, welche sich im zwischenmenschlichen Kontakt im Rahmen der Arzt-Patienten-Beziehung ergeben.

Wie die Autoren schreiben, wurde diese «vertragsbedingte Kondition nicht von medizinischen Gutachtern» erdacht. Wäre es nicht Aufgabe aller Mediziner, vermittelnd zu wirken? Die psychiatrischen Methoden des Erkenntnisgewinns sollten innerhalb des Medizinalsystems adäquat gewichtet werden. Es deutet einen Rückfall in dunkle Zeiten an, wenn kranke Menschen als unsere Gesellschaft aussaugende Monster dargestellt werden.

Dass nun das Bundesgericht sogar den Nachweis der Therapieresistenz fordert (vergl. Medienberichte vom 17.7.2017 SRF), verdeutlicht die Groteske. Viele Psychiater/-innen erachten auch Patienten mit einer IV-Rente nicht als austherapiert. Die Rente kann eine Grundlage schaffen, damit genügend Ruhe und Sicherheit entsteht, damit überhaupt eine Verbesserung der Situation wieder in kleinen Schritten und über mehrere Monate oder Jahre entstehen kann. Dass das Instrument Rentenrevision gut etabliert ist und somit Verbesserungen des Gesundheitszustandes wiederum erfasst werden könnten, scheint vergessen gegangen zu sein.

Dr. med. Maria Cerletti Fachärztin für Psychiatrie und Psychotherapie, FMH

1 Felix Schneuwly wechselt zu comparis.ch. Medienmitteilung vom 23.11.2011.

2 Brühlmeier Rosenthal D. Soziales Elend nach Stopp oder Verweigerung von IV-Renten. Schweiz Ärztezeitung. 2017;98(24):785-7.

3 Lüthi T, Amrein M. Russe will das menschliche Hirn vermessen. NZZ am Sonntag vom 14.7.2017.

\section{Verständnis für die Versicherungs-}

\section{mediziner}

Brief zu Mast H., Schneuwly F. Mehr Verständnis für die eidgenössische Versicherungsmedizin. Schweiz Ärztezeitung 2017;98(28-29):918-21.

Ob Prof. Mast mit seinem "Habt uns doch bitte auch lieb»-Artikel mehr zum Verständnis beigetragen hat, wage ich zu bezweifeln. Der Unterschied zwischen einem Gutachter für die IV und einem Behandler ist nicht die Frage ob er pro bono arbeitet, sondern die Arbeit muss sich rentieren. Die Rechung ist ganz einfach. Für eine Patientenstunde kann ich rund 180 Franken abrechnen, für ein monodisziplinäres IV-GA bietet die IV rund 3200 Franken. D.h. ich muss es in 17,7 Stunden fertig haben damit es sich «lohnt» (nicht zu reden von den Stundenansätzen in der Forensik). Der Effekt ist, dass Aktenauszüge von der Sekretärin oder per unkritischem Copy-andpaste gemacht werden und die Untersuchung im psychiatrischen Teil kaum mehr als eine Stunde dauert, damit es sich "rentiert». Im sozialrechtlichen Gutachterwesen zeigt sich exemplarisch, was Gesundheits «ökonomie» bedeutet. Wer also gewissenhaft und umfassend begutachten möchte, zahlt nicht selten drauf. Unabhängig kann nur bleiben, wer verschiedene Auftraggeber hat, inkl. Patient.

Die Frage stellt sich, ob es für die Sachbearbeiter und den RAD unangenehmer ist, in einem gewissen Prozentsatz Klagen der Behandler und Anwälte zu bearbeiten oder die Rückfragen des eigenen Rechtsdienstes und des BSV. Der ethisch-moralische Aspekt besteht aus meiner Sicht in der Begegnung mit dem Versicherten, der in der Regel schon sehr lange aus einem Arbeitsprozess herausgefallen ist. Leider kommt es nicht selten vor, dass aufgrund des beschriebenen Zeitdruckes die Menschen nicht angehört werden und auch nicht versucht wird zu verstehen, wie es zu dem Leiden gekommen ist und warum sie sich nicht mehr arbeitsfähig fühlen. Vielleicht sind sie arbeitsfähig. Sie haben aber sicher ein Recht genau und gewissenhaft untersucht zu werden, mit möglichen Differentialdiagnosen und gegebenenfalls weiterführenden Abklärungen. Das wiederum braucht Zeit, aber vor allem Interesse an dem Gegenüber. Versuchen Sie einmal eine Arbeitsfähigkeit ohne ICD-10 Diagnose zu begründen. $\mathrm{Ob}$ das wirklich sekundär ist? Alle, die zum Gutachten kommen, haben bereits vorgängig Dignosen von ihren Behandlern bekommen (müssen) - was das mit überwiegender Wahrscheinlichkeit zu tun hat, bleibt mir ein Rätsel. Dass diese Diagnosen kritisch gewürdigt und diskutiert werden müssen, ist klar. Wenn aber ein Gutachten zum Schluss kommt, die Person leidet unter keiner Diagnose, ist es doch eine Frage der Zeit bis eine Krankenkasse die weiteren Leistungen verweigert.

Dass eine Gutachter nicht behandeln sollte, ist Basiswissen. Der Explorand ist aber in keiner Eignungsprüfung für was auch immer, sondern in einer gewissenhaften ärztlichen Untersuchung. Dafür haben wir den gefragten Sachverstand. Eignungsprüfungen können sonst auch Sachbeabeiter machen oder die Akten können auch Sekretärinnen «sachverständig» lesen. Anders als im forensischen Kontext sitzen uns hier Menschen gegenüber, die auch Patienten sind. Im Strafverfahren haben viele keine Diagnose.
Es ist richtig, dass jeder erfahrene Kliniker mit versicherungsmedizinischem Basiswissen auch ein guter Gutachter sein kann. Der Umkehrschluss stimmt aber aus meiner Sicht nicht, weil es doch eine Reihe von Gutachtern gibt, die nicht mehr oder nur sehr wenig im Alltag mit Patienten im Kontakt sind und damit das Gespür für diese Menschen verlieren.

Als Gutachter ist man nicht Anwalt einer Seite. Als Arzt sollte man sich aber seiner ethische Verantwortung bewusst sein. Es ist doch traurig, dass Juristen, nicht Ärzte, als Patientenanwälte die Päusbonog gekippt haben.

Dr. med. Christoph Burz, Summaprada

\section{Unverständliche Aktion gegen Ignazio Cassis}

Die unselige Plakataktion der Zürcher Ärzte in Bern (s. NZZ vom 20. Juli 2017 «Die Zürcher Ärzte schiessen scharf gegen Cassis») wird ihr Ziel in mehrfacher Sicht verfehlen, denn es ist ein Schuss auf den Mann statt auf die Sache. Wer soll denn diese Aktion verstehen? Was soll sie bringen? Für die Bevölkerung ist schwer verständlich, wie sich die grossen Einkommensunterschiede bei den Ärzten erklären, warum gewisse Eingriffe immer noch so teuer sind, auch wenn sie schneller und einfacher durchführbar geworden sind und warum die Prämien weiter steigen. Sehr wenig Verständnis hat der Bürger wohl auch für die unrühmliche Zerstrittenheit der Ärzteschaft, was TARMED betrifft. Und nun "schiesst» eine kantonale Gesellschaft auch gleich noch gegen einen Kollegen, der für den Bundesrat kandidiert. Glauben die Zürcher Ärztinnen und Ärzte allen Ernstes, dass ein anderer Bundesratskandidat ihre Anliegen besser vertreten wird? Dass eine andere Kandidatin ohne so viel Erfahrung im Gesundheitswesen auf wundersame Weise das Problem der steigenden Kosten in den Griff bekommt und die vielen (divergierenden) Anliegen der zerstrittenen Ärzte elegant löst? Was können wir denn mehr wollen, als einen Arzt im Bundesrat, der die Arbeit der Kantone kennt, der im Bundesparlament Erfahrung hat, der ausgewogen in der Mitte politisiert, aber auch nach Rechts und Links zuhören kann, der alle Landessprachen fliessend spricht, der die (berechtigten) Sorgen der Krankenkassen kennt, den wir selbst mit grosser Begeisterung in den FMH-Vorstand gewählt haben, der kritisch denkt und seine Meinung verteidigt, auch wenn er gegen die Strömung argumentieren muss (man nennt das Standfestigkeit).

Mir ist diese populistische, pseudo-neutrale Aktion der Zürcher Kollegen sauer aufgestos- 
sen. Die Kommentare, die ich in meinem Umfeld dazu gehört habe, haben mich beschämt. Es fehlt nur noch der Twitter, und wir sind in den USA. Das Plakat wird nicht als konstruktiver Beitrag der Ärzteschaft zur Lösung eines grossen Problems wahrgenommen, sondern als «Werbegag» in eigener Sache. Die Aufgabe von uns Ärztinnen und Ärzten ist es aber, Wege der Verbesserung zu suchen und zu finden, Kompromisse einzugehen, vielleicht auch innovative Ansätze zu entwickeln. Scharmützel auf diesem Niveau schaden der ganzen Berufsgruppe und bringen uns nicht weiter. Warum sind denn die Ärzte zerstritten? Doch nur, weil die einen (vermeintlich) zu viel und die anderen im Vergleich zu wenig verdienen, während die Kosten stark steigen. Ignazio Cassis ist Arzt, der auch die Seite der Patienten versteht (um die geht es im Gesundheitswesen ja auch ...). Er ist ein erfahrener Politiker, der auch die Anliegen der Ärzte bestens nachvollziehen kann. Aber auch er wird Kompromisse vertreten müssen und er wird unpopuläre Entscheide mittragen müssen. Ignazio Cassis wird mit seiner Persönlichkeit und seiner Erfahrung dem Gesundheitswesen sehr gut tun und er wird auch unsere Anliegen gut vertreten (soweit es ein «Unsere» gibt). Es wäre wirklich wichtig, dass wir die einmalige Chance, einen kompetenten, klugen Kollegen im Bundesrat zu haben, mit solchen Aktionen nicht vergeigen.

Dr. med. Silva Keberle, Basel

\section{Communications}

\section{Korrigendum}

In der deutschen Fassung des Artikels Praxisassistenz von François Pilet und Bernard Giorgis [1] wurde der französische Ausdruck «formation postgraduée» bzw. «formation» fälschlicherweise mit «Nachdiplomausbildung» bzw. «Ausbildung» übersetzt. Gemeint ist in diesem Kontext die Weiterbildung der Hausärztinnen und Hausärzte. Die Autoren bedauern, dass dieser Fehler bei der Überprüfung der Übersetzung übersehen wurde.

1 Giorgis B, Pilet F. Praxisassistenz. Schweiz Ärztezeitung 2017; 98(24):765-6.

\section{Sujet d'actualité en ligne -} www.bullmed.ch/fr/tour-dhorizon
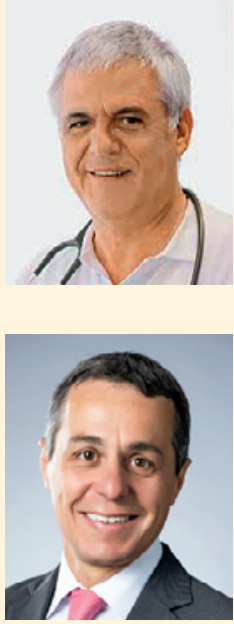

Dr méd. Josef Widler, président de la société de médecine du canton de Zurich

\section{Pseudo-solution populiste}

Economiser sur le dos des médecins - un mauvais calcul!

Interview d'Ignazio Cassis, conseiller national (PLR) et médecin

Révolution ou simple tempête dans un verre d'eau?

La CSSS veut des budgets globaux et un accord sur le TARMED, si besoin par la contrainte 